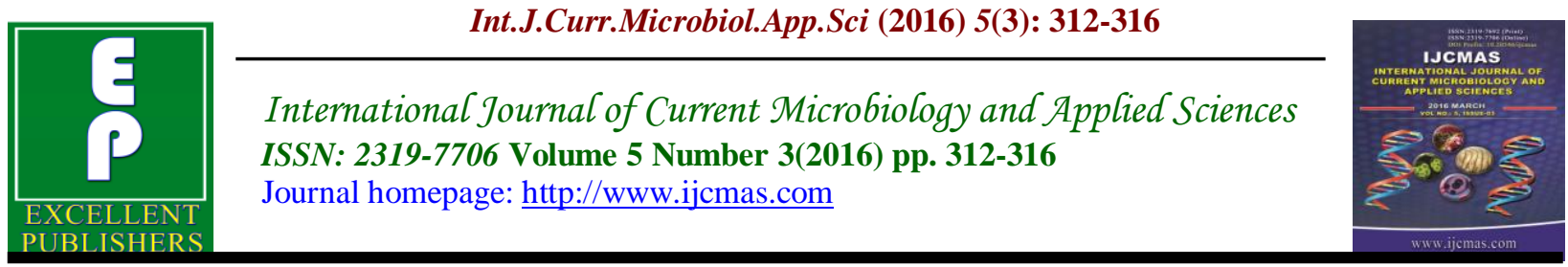

Case Study

http://dx.doi.org/10.20546/ijcmas.2016.503.038

\title{
Acute Intermittent Porphyria: A Rare Inborn Error of Heme Metabolism
}

\author{
Nabeel Mushtaque Ahmed*, Vinay Pandey and Ashish Srivastava
}

Golden Sands Apartments, Sir Syed Nagar, Aligarh, Uttar Pradesh, India

*Corresponding author

Keywords

Inborn Error of

Heme Metabolism, Intermittent, Porphyria.

\section{Article Info}

Accepted:

15 February 2016

Available Online:

10, March 2016
A B S T R A C T

A 22 year old young female presented with recurrent abdominal pain, generalized weakness and wasting with hypertension in Medicine OPD of JNMC. Subsequently she was diagnosed as a case of Acute Intermittent Porphyria (AIP). Though not common, any patient with unexplained abdominal crisis specially young with any peripheral or central nervous system dysfunction should raise the clinician's suspicion of being porphyric patient as prompt management can relieve the patient from progressive neurological damage and disability.

\section{Introduction}

Porphyrias are disorder of haem biosynthetic pathway. The one with most serious consequences is acute intermittent porphyria (AIP), inherited as an autosomal dominant disease, remains clinically silent in the majority of the population who carry the trait. Presentation is in early adult life usually around the age of 30yrs and women are more affected than male. The disease is widespread but specially common in Scandinavia \& Great Britain. The disorder is caused by partial deficiency of porphobilinogen deaminase enzyme, leading to increased excretion of Aminolevulinic Acid (ALA) and Porphobilinogen (PBG) in the urine (Fauci, et al.; Rajashekar Reddy, et al., 2002). The presentation is an acute relapsing and remitting neurological syndrome, invariably with acute abdominal pain and features of autonomic dysfunction such as tachycardia, hypertension, and constipation. Other frequent associations include neuropsychiatric manifestations like depression, anxiety, psychosis and convulsions. Often there is profound hyponatraemia due to inappropriate $\mathrm{ADH}$ release. Neuropathy is predominantly motor (70\%) \& can lead to quadriplegia with respiratory muscle paralysis. Cranial \& sensory nerves may be affected. Skin is never affected like other photosensitive porphyria.

There is no proven explanation for the episodic pattern of these attacks. However they are often provoked by drugs, diet, alcohol and even fasting. In a significant number of cases no precipitant can be identified (Boon Nicholas, et al.; Chong, et 
al., 1978). The diagnosis can be confirmed by demonstrating an increased amount of PBG in the urine during an attack. Freshly voided urine is of normal colour but it may turn dark upon standing in light and air. All family members should be screened to detect latent cases (Kumar Parveen and Clark Michael; Fauci, et al.,). Treatment with a high carbohydrate diet diminishes the number of attack in some patients. Acute attacks may be life threatening and require prompt diagnosis and withdrawal of the inciting agent and treatment with narcotic analgesic and intravenous high conc. glucose and haem arginate which inhibits ALA synthase activity and thus reduce ALA and PBG level. The intravenous dose of haem is up to $4 \mathrm{mg} / \mathrm{kg}$ twice daily for four days keeping in mind the adverse consequences especially phlebitis \& coagulopathy. Recovery from severe motor neuropathy may require long time even years. Liver transplantation is an option for patients with disease poorly controlled by medical therapy (Fauci, et al.; Boon Nicholas, et al.,).

\section{Case Report}

A 22 year old female, hailing from Hathras, was admitted in Medicine ward JNMC with the complaints of episodic attacks of severe abdominal pain for the last 3 years, associated with generalized weakness, wasting and walking difficulties for the same duration. Patient elaborated that pain was diffuse, not associated with fever or vomiting but aggravated by fasting and unrelieved by antacids. She had no preceding NSAID intake history, or any history of previous jaundice, diabetes or hypertension. She admitted for several times during relapse of pain but the diagnosis was inconclusive though she improved after symptomatic treatment. There was no history of trauma, sexual exposure, contact with chemicals or alcohol abuse. She had no bladder or bowel difficulty except constipation during attack. Her appetite was good and she was not suffering from any respiratory or cardiac disease. No family member was suffering from such illness. On examination, patient was found conscious ill looking, malnourished, mourning with pain, dehydrated, non anaemic \& non icteric. No cyanosis, clubbing or pigmentation was found. Her temperature was 99F, RR 16/minute, no enlarged lymph nodes found. Thyroid gland was normal, Pulse 140/m, regular \& BP 170/100 $\mathrm{mmHg}$ in supine position and $140 / 90 \mathrm{mmHg}$ in standing position. Bedside urine colour was normal on fresh sample but later on it became redtea colour.

Abdominal examination showed diffuse tenderness on deep palpation but no hepatosplenomegaly, ascites or visible veins was found. Hernial orifices were intact. Genitourinary system examination revealed no abnormality. On neurological examination higher cerebral function was found normal, no cranial nerve palsy was seen. Wasting of muscles of both upper and lower limbs was present with hypotonia. Muscle power diminished in both limbs $(3 / 5)$ in all groups. Superficial reflexes were present but deep reflexes were diminished in both upper and lower limbs, more marked distally, Plantar reflexes were flexor, gait was high stepping, no tremor or involuntary movement was present. No sensory abnormality was detected; vibration and joint position sense were normal, Romberg's sign was negative. No peripheral nerve thickening or no sign of meningeal irritation found.

\section{Laboratory Profile}

$\mathrm{Hb} \%$ II gm|dL,ESR $15 \mathrm{~mm}$ in 1st hr. TLC $8.5 \times 10^{\wedge} / \mathrm{L}$. Platelet $220 \times 10^{\wedge} 9 / \mathrm{L}$, Urine R/E: Sugar-Nil. Albumin-Nil, Pus cells - 02|HPF, RBC Nil RBS: 85 mg/dl, Peripheral 
blood film: Eosinophilia. Serum Na+ 128 mmo $1 / \mathrm{L}$, creatinine $0.7 \mathrm{mg} / \mathrm{dl}$ Bilirubin 0.3 $\mathrm{Mg} / \mathrm{L}, \quad \mathrm{HBsAg}$ negative.SGPT 89 UL, Serum amylase $79 \mathrm{U} / \mathrm{L}$. TSH:2.51 mU/L, VDRL: Non reactive

$\mathrm{X}$ ray chest P/A Clear, ECG showed Sinus Tachycardia. Plain X-ray Abdomen A/P normal,USG Whole Abdomen,Endoscopy of upper GIT and Echocardiography all were normal. Slit skin smear from ear \& forehead: negative for mycobacterium leprae. NCV: Motor polyneuropathy.

\section{Urine for Porphobilinogen: PBG Positive}

Thus the diagnosis was established as a case of an acute intermittent porphyria. The
Patient had a stormy course in hospital with recurrent severe colicky abdominal pain which was controlled by intravenous opioid analgesics. Dehydration and hyponatremia were corrected by 5\% Dextrose Saline infusion. There was labile hypertension and tachycardia which was controlled by propranolol. 25\% glucose infusion \& plenty oral glucose rich fluid intake helped in subsiding episodes of severe pain. The patient was discharged after a period of 20 days hospital stay. She was given a chart of harmful drugs that may precipitate the condition. During discharge her condition was stable, neurological deficit was significantly improved and hypertension was controlled. Her family members were advised to screen for urine porphobilinogen.

Figure.1 There is Sample of Freshly Voided Urine of Normal Colour (Left Side) and Dark Reddish Urine (Right Side) After Exposure to Sunlight

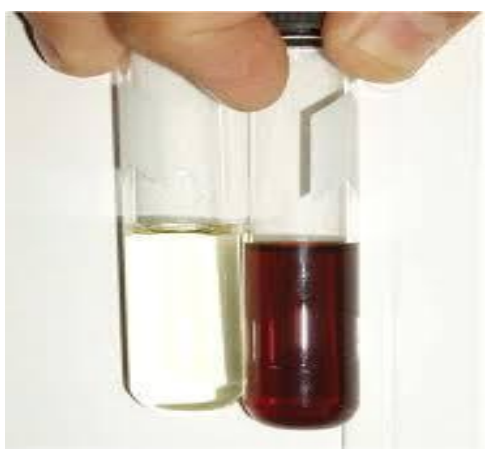

Figure.2 Biosynthetic Pathway of Heme

\section{HEME BIOSYNTHETIC PATHUAY}

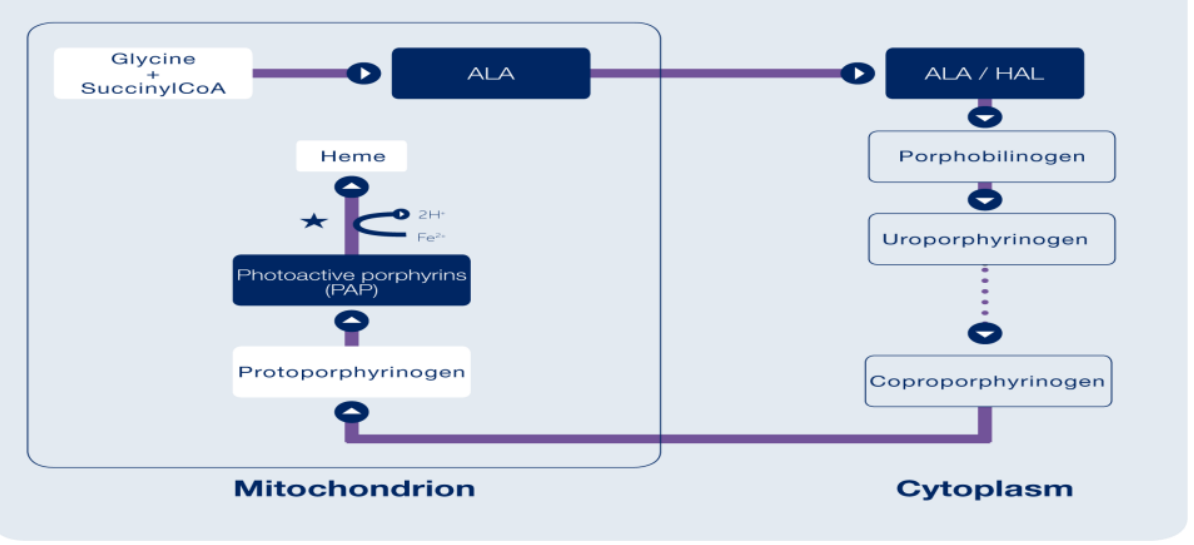


An acute attack of AIP varies considerably in its clinical presentation and patients may be referred to an internist, surgeon, neurologist, psychiatrist or gynaecologist. A high level of clinical suspicion is necessary in order not to miss the diagnosis which is based on relatively simple laboratory examination for porphobilinogen. An increased urinary excretion of porphobilinogen in the absence of lead poisoning points towards a diagnosis of acute intermittent porphyria. Our patients did not have any history of contact with lead substances. Moreover no clinical feature of lead poisoning like bluish discoloration of gums due to lead sulfide deposition (burton's line) or Basophilic stippling in red blood cells was absent ${ }^{4,5}$. A brief review of the haeme biosynthetic pathway is outlined in order to explain the fundamental defect in AIP.

ALA synthetase is the first and also the rate limiting enzyme in the pathway. In AIP there is reduction in the enzyme $\mathrm{PBG}$ deaminase, resulting in diminished haem synthesis causes induction of the rate limiting enzyme ALA synthetase via a biofeedback mechanism and thus results in high level porphobilinogen(PBG) in the blood and urine. PBG is generally so strikingly increased during an attack of AIP, quantitative event on spot sample rather than a 24 hour collection of urine is highly informative. During collection of urine a dark plastic jug should be used to protect the sample from light as porphyrin are fluorescent substances which on exposure to light and air oxidize and turn intensely reddish. During shipping it is stored in a refrigerator or in an ice chest (Fauci, et al.; Chong, et al., 1978). The diagnosis of AIP is always an important item of medical information even when there is no symptom. It may influence the choice of drugs to treat other medical conditions, surgery and anesthesia. Some drugs are potentially dangerous like sulphonamide, Tranquilizers and sedative, Griseofulvin, Carbamazepine, Phenytoin, Valproate, OCP, Alcohol, Rifampicin, Tetracycline, Diclofenac. Acute attacks require a prompt diagnosis, withdrawal of the inciting agent, and treatment with narcotic analgesics, intravenous glucose and haematin. Electrolyte imbalances requires careful monitoring and correction. Treatment with a high carbohydrate diet diminishes the number of attack in some patients. A minimum of $300 \mathrm{gm} /$ day of carbohydrate should be provided orally or intravenously. Heme products like haeme arginate, is the recommended specific therapy for AIP and should be started as early as possible; the administration of this end-product of the haem biosynthesis pathway improves the underlying biochemical disturbance by reducing porphyrin precursor excretion. Adverse effects commonly seen are phlebitis and coagulopathy. This costly drug is not routinely available in our country. So management strategies for this rare disease should be planned to relieve symptoms, prevent inciting factor, avoiding harmful drugs and taking plenty glucose rich fluids regularly in our perspective (Fauci, et al.; Boon Nicholas, et al.; Becker and Kramer, 1977.).

\section{References}

Becker, D.M., Kramer, S. 1977. The neurological manifestations of porphyria. A Rev. Med., 56: 411423.

Boon Nicholas, Colledge Nicki, Worker Brian, Hunter John. Davidson's Principles and Practice of Medicine. 21st edition, Page 456-458.

Chong, K.F., Bin Omar, A.R., Rajab, S. 1978. Acute Intermittent Porphyria A case report. Singapore Med. J. 19. 
Fauci, Braunwald, Kasper, et al., Harrison's Principles of Internal Medicine. 17th edition, Page 2434-2443.

Kumar Parveen, Clark Michael. Clinical Medicine. 6th edition, Page 1150.

Rajashekar Reddy, Nitin, K., Sethi, Ish Anand, P.K., Sethi. 2002. Acute Intermittent Porphyria. Management Aspects-JIACM, 3: 252-7.
Tschudy, D.P., Valsamis, Magnussen. 1975. Acute Intermittent Porphyria. Clinical \& Selected Research Aspect. Ann. Int. Med., 83: 851-864.

Vembu Periasamy, Asmahan Al Shubaily Girsh. 2002. Diagnostic Dilemmas in Acute Intermittent Porphyria, a case report. Med. Principles and Practice, 11.

\section{How to cite this article:}

Nabeel Mushtaque Ahmed, Vinay Pandey and Ashish Srivastava. 2016. Acute Intermittent Porphyria: A Rare Inborn Error of Heme Metabolism. Int.J.Curr.Microbiol.App.Sci. 5(3): 312316. doi: http://dx.doi.org/10.20546/ijcmas.2016.503.038 